\title{
Refractive optics using lithium metal
}

\author{
D. A. Arms, ${ }^{\text {a) }}$ E. M. Dufresne, R. Clarke, and S. B. Dierker ${ }^{\text {b) }}$ \\ Department of Physics and MHATT-CAT, University of Michigan, Ann Arbor, Michigan 48109-1120 \\ N. R. Pereira \\ Ecopulse Inc., P.O. Box 528, Springfield, Virginia 22150 \\ D. Foster \\ Army Research Laboratory, Adelphi, Maryland 20873
}

(Presented on 23 August 2001)

\begin{abstract}
Thanks to its low x-ray absorption, lithium should be the material of choice for x-ray refractive lenses. This article discusses some of the measurements done to verify lithium's relevant properties. Both $\mathrm{x}$-ray transmission and refraction are consistent with expectations. The lens gain suffers from broadening that is related to small-angle scattering. (C) 2002 American Institute of Physics.
\end{abstract} [DOI: $10.1063 / 1.1436547$ ]

Since the re-evaluation of refraction for x-ray optics in the context of synchrotron radiation about 1 decade ago, ${ }^{1-3}$ various groups ${ }^{4-7}$ have shown that refractive $\mathrm{x}$-ray lenses can be quite useful. They are presently in use at several synchrotrons, and the x-ray study presented here was similarly done at the Advanced Photon Source with the 7-ID undulator line operated by the University of Michigan, Howard University, Lucent Technology-Bell Labs Collaborative Access Team (MHATT-CAT).

For $x$ rays, the index of refraction is less than unity, ${ }^{8}$ so that a lens that focuses $x$ rays is concave. The index of refraction difference with unity,

$$
\delta=n-1,
$$

is very small: for materials and $\mathrm{x}$-ray energies of interest, $\delta$ is $10^{-5}-10^{-6}$. For refractive optics, the focal length $f$ of a biconcave lens with radius of curvature $R$ is

$$
f=\frac{R}{2 \delta} \text {. }
$$

For a single $\mathrm{x}$-ray lens with a macroscopic radius, such as $R=0.2 \mathrm{~mm}$ used by Lengeler et al. ${ }^{5}$ the focal length is then even longer than most synchrotron beam lines. For example, at $\mathrm{keV}$ where $\delta=0.96 \times 10^{-6}$ for lithium, such a lens made from lithium has a focal length of $f=100 \mathrm{~m}$.

The focal length becomes smaller by refocusing the radiation with additional lenses. Using a large number of lenses, with $N \sim 10-100$, the resulting compound refractive lens ${ }^{4,5}$ has a focal length that is reduced to $1 \mathrm{~m}$ or so. Such lenses bring typical applications of conventional optics, including collimation, microfocusing and microscopy, into the $\mathrm{x}$-ray regime.

To date, the most successful $\mathrm{x}$-ray lenses have been made with conventional materials such as aluminum and plastics, as lenses made with these materials perform closest

${ }^{a)}$ Electronic mail: dohnarms@anl.gov

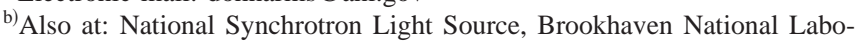
ratory, Upton, NY 11973-5000. to their theoretical capabilities. The reason is simply welldeveloped manufacturing techniques. For the same reason, lenses made with silicon work quite well, especially for focusing in a single dimension when quite sophisticated lens profiles can be conveniently made by deep etching and related lithography techniques. ${ }^{9,10}$

Aluminum and plastics are, unfortunately, not the best materials for this application. For refractive optics the figure of merit ${ }^{3}$ is the phase shift per attenuation length. This favors low atomic number materials. Beryllium is an obvious candidate, but it seems that conventional grades of beryllium suffer from excessive scattering of $\mathrm{x}$ rays or other problems that make it difficult (and expensive) to produce good beryllium $\mathrm{x}$-ray lenses.

To achieve optimum performance from refractive $\mathrm{x}$-ray optics, we are developing lenses from lithium. Lithium transmits $x$ rays $2-3$ times better than beryllium. Even so, lithium is not often used for $\mathrm{x}$-ray optics because of its reputation as a difficult and dangerous material. Large quantities of lithium under the wrong circumstances can indeed result in serious problems, such as explosions. However, an X-ray lens needs only a few grams of lithium. Lithium is of course stable in an inert enviroment such as vacuum, and since much x-ray work takes place in a vacuum, the two are perfectly compatible. Coating lithium with a thin $(0.3 \mu \mathrm{m})$ layer of parylene ${ }^{11}$ makes it possible to handle lithium in open air, for a short time at least.

For ease of manufacturing, we test lithium's performance in a refractive $\mathrm{x}$-ray lens with Cederström's alligator lens geometry. ${ }^{12}$ This one-dimensional lens is basically a series of prisms at an angle $\theta$ with the x-ray beam, as shown in Fig. 1. Manufacturing convenience strongly favors $90^{\circ}$ for the prism's top angle. A tooth of height $h$ is then separated from its neighbor by $2 h$, and the number of teeth in a lens is given by $N=L / 2 h$, where $L$ is the length of the lens. All of our lenses have a length of $L=111 \mathrm{~mm}$ and are $6 \mathrm{~mm}$ wide. Lens prototypes have been made with different tooth heights, with $h$ ranging between 0.15 and $1.5 \mathrm{~mm}$.

This article shows some of the measurements needed to 


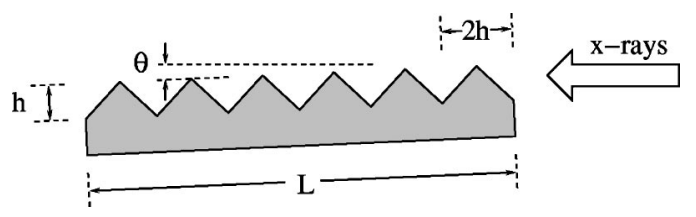

FIG. 1. Half of a lithium lens, represented by a single jaw, as used for testing. A full lens would have a second inverted jaw above the first at the same angle $\theta$. The height of each tooth is $h$, the lens pitch is $2 h$, and the lens length is $L$.

characterize lenses whose performance has already been presented elsewhere. ${ }^{13} \mathrm{X}$-ray results obtained to date include a factor of 3 gain, that is, a threefold increase in intensity over an unfocused beam. Although useful, this gain is less than should be theoretically possible. We believe this is due to manufacturing defects, and not to any fundamental problems with lithium.

Figure 2 shows focusing by one jaw of a prototype lens. This jaw has 74 teeth with a height of $h=0.75 \mathrm{~mm}$. It sits $200 \mathrm{~mm}$ behind a $0.7 \mathrm{~mm}$ wide slit that is $49 \mathrm{~m}$ away from the x-ray source. The energy of the photons is set to $10 \mathrm{keV}$ by a silicon monochromator. The image is taken with a charge coupled device (CCD) camera $7 \mathrm{~m}$ behind the lens.

The unfocused beam has wings caused by penumbra, and a profile that approximates a centered cut of a Gaussian beam. The peak intensity in the focused beam is 2.5 times larger than the unfocused beam, a modest gain. Assuming Gaussian optics and a Gaussian source, while taking into account lithium's absorption, the theoretical gain is 5.2.

The principal reason for the twofold discrepancy is that the focal spot is almost twice as wide as expected. In addition, the beam shape distinctly shows an undesirable shoulder (see Fig. 2). These nonideal features are not yet understood in detail; some small angle scattering from $\sim 1 \mu \mathrm{m}$ surface imperfections is expected, but not yet modeled. In addition there is contamination by a small $30 \mathrm{keV}$ component of the beam as discussed later.

To test the quality of the teeth we measure the transmission of a thin $(30 \mu \mathrm{m})$ slice of the X-ray beam through all $N$

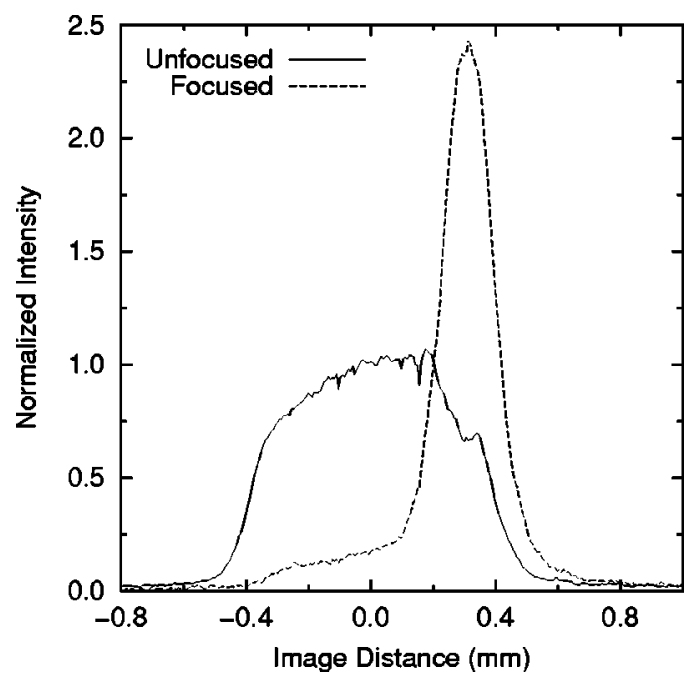

FIG. 2. Focusing by one jaw of a lithium lens. The focused beam has a maximum 2.5 times larger than the unfocused beam, half the predicted factor of 5.2.

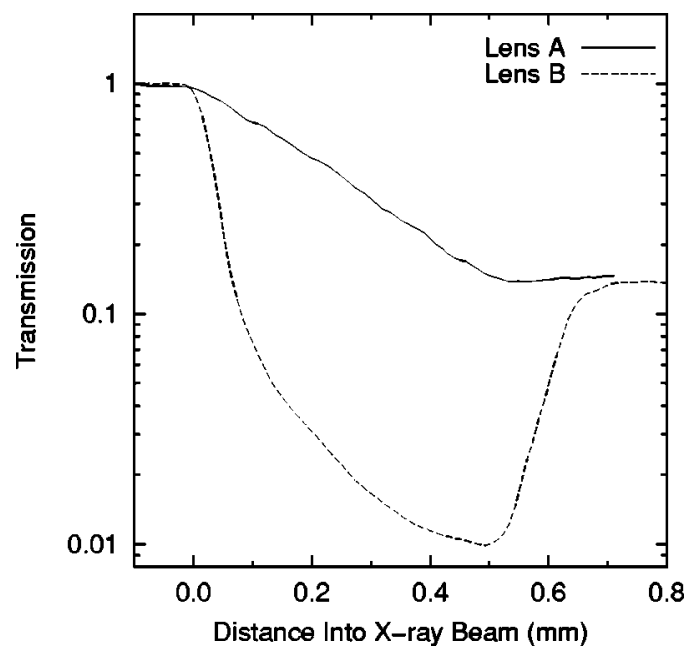

FIG. 3. Transmission of a $30 \mu \mathrm{m}$ beam through a jaw as it is inserted into the beam, while held parallel to the beam. Lens $A$ is handled properly, in dry nitrogen and then under a constant vacuum. Lens $B$ 's transmission decreases 1 order of magnitude faster, due to corrosion from water vapor that was introduced by a leaky seal.

teeth, when $\theta$ is set to zero. The absorption can be easily measured by collecting the transmitted flux with an ionization chamber. When the beam passes above the top of the teeth, there is absorption from only the $0.125 \mathrm{~mm}$ beryllium entrance and exit windows (which is basically negligible), making this the maximum transmission. When the beam reaches the bottom of the teeth, it passes through $111 \mathrm{~mm}$ of lithium. Inbetween, there should be an exponential decrease of the intensity. Any deviation betrays a problem with the material, the tooth surface, or something similar.

Figure 3 shows the $\mathrm{x}$-ray transmission for two identical lenses with $0.5 \mathrm{~mm}$ high teeth (different from the lenses shown earlier). Lens $A$ was kept in a good vacuum, while lens $B$ was found to have been kept in a vessel with a leaky seal for several days in humid air. As lens $A$ is inserted into the beam, the amount of lithium traversed by the x-ray beam is approximately linear. The exponential decrease in transmission is evident in the linear slope on the semilog plot. After $0.5 \mathrm{~mm}$ the beam reaches the bottom of the $0.5 \mathrm{~mm}$ high teeth, and the transmission dips slightly. Any further insertion has the beam passing though the $111 \mathrm{~mm}$ bulk of the lithium, so that the transmission remains the same. The transmission agrees with lithium's theoretical absorption length of $58 \mathrm{~mm}$ at $10 \mathrm{keV}$. Lens $B$ was highly corroded due to water vapor, and the transmission through the teeth is roughly a factor of 10 worse due to the presence of oxygen on the surface. Once the insertion reaches $0.5 \mathrm{~mm}$, the transmission rises to the transmission seen by lens $A$; apparently, the corrosion only affects lithium's surface, not its bulk.

A CCD camera $8 \mathrm{~m}$ behind the ionization chamber measured the beam's deflection. Figure 4 shows slices of the images, when the lens is both out of the beam and in the beam. The beam profile without the lens shows a single peak with a full width half maximum (FWHM) of $14 \mu \mathrm{rad}$ that corresponds to the slit width modified by the beam's native divergence. However, the beam that passed through the lens shows two peaks. The principal peak, deflected by $175 \mu \mathrm{rad}$, 


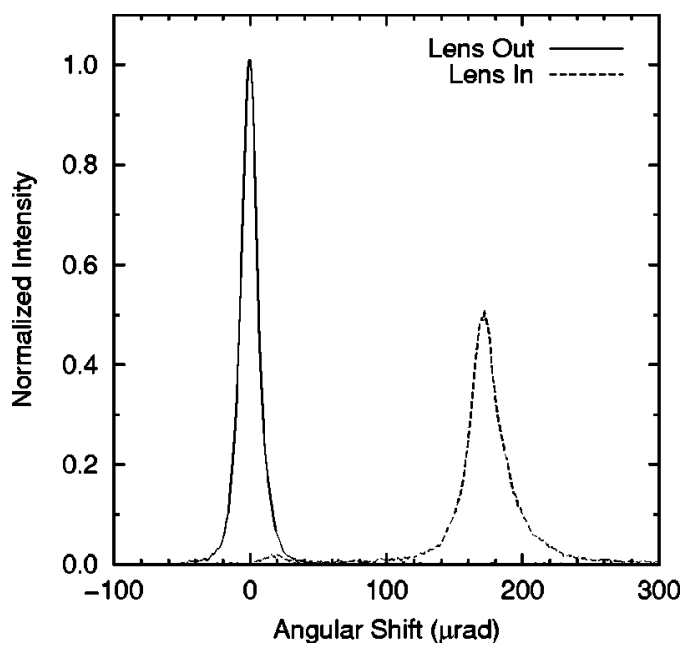

FIG. 4. A slice through a CCD image before and after the lens is inserted into the $30 \mu \mathrm{m}$ beam. The large peak for when the lens is in the beam is due to the first harmonic $10 \mathrm{keV}$ photons, while the tiny peak is due to the third harmonic $30 \mathrm{keV}$ photons.

has a FWHM of $25 \mu \mathrm{rad}$. There is also a small secondary peak, with a deflection of $15 \mu \mathrm{rad}$. The first peak corresponds to the $10 \mathrm{keV}$ photons passed by the monochromator (using Si 111). However, the monochromator also passes 30 $\mathrm{keV}$ third-harmonic photons (from Si 333), corresponding to the second peak. The roughly ninefold smaller deflection of the small peak compared to the large peak agrees with the quadratic decrease of $\delta \propto 1 /(h \nu)^{2}$ with threefold increasing photon energy $h \nu$. The nearly doubling of the FWHM values for the peaks is to be investigated further.

The deflection of the $10 \mathrm{keV}$ photons allows a measurement of the index of refraction decrement $\delta$. For a single right-angle prism (a single tooth), the deflection $\Delta \theta$ due to the front and back surface is $2 \delta$. For $N$ teeth, the deflection is

$$
\Delta \theta=2 N \delta \text {. }
$$

So for a jaw of $N=74$ ideal teeth and a measured deflection of the beam of $175 \mu \mathrm{rad}$, the resulting $\delta$ is $1.15 \times 10^{-6}$. This value is roughly $20 \%$ larger than the expected value of $0.96 \times 10^{-6}$. At the present precision of the measurements, the discrepancy between the deflection values is not serious.

A refractive lens can also be used for filtering higher harmonic photons along with focusing, when it is used in combination with an aperture passing only the focused beam. The higher harmonic photons refract less so that they con- verge much farther away than the primary focus, allowing them to be blocked by the aperture.

The measurements of transmission and refraction agree quite satisfactorily with the values found in the literature. Clearly, lithium is transparent enough to pass most of the beam's $\mathrm{x}$ rays. However, the divergence of the $\mathrm{x}$ rays after the lens is almost double the divergence of the $\mathrm{x}$ rays before the lens, leading to a wider $\mathrm{x}$-ray spot and a degraded focus that limits the intensity gain. Whether this excessive divergence can be suppressed by more precise manufacturing remains to be seen.

Operation of the MHATT-CAT Sector 7 beamlines at the Advanced Photon Source is supported by DOE Grant No. DE-FG02-99ER45743. Use of the Advanced Photon Source is supported by the U.S. Department of Energy, Basic Energy Sciences, Office of Energy Research, under Contract No. W-31-109-ENG-38. N. R. P. is supported by a Phase I SBIR award from BMDO.

${ }^{1}$ S. Suehiro, H. Miyaji, and H. Hayashi, Nature (London) 352, 385 (1991).

${ }^{2}$ A. G. Michette, Optical Systems For X-rays (Plenum, New York, 1986); A. G. Michette, Nature (London) 353, 510 (1991).

${ }^{3}$ B. X. Yang, Nucl. Instrum. Methods Phys. Res. A 328, 578 (1993).

${ }^{4}$ A. Snigirev, V. Kohn, A. Snigireva, and B. Lengeler, Nature (London) 384, 49 (1996).

${ }^{5}$ B. Lengeler, J. Tuemmler, A. Snigirev, I. Snigireva, and C. Raven, J. Appl. Phys. 84, 5855 (1998); B. Lengeler, C. Schroer, J. Tuemmler, B. Benner, M. Richwin, A. Snigirev, I. Snigireva, and M. Drakopoulos, J. Synchrotron Radiat. 6, 1153 (1999). Other articles are available at http:// www.xray-lens.de

${ }^{6}$ H. R. Beguiristain, M. A. Piestrup, R. H. Pantell, C. K. Gary, J. T. Cremer, and R. Tatchyn, Synchrotron Radiation Instrumentation: Eleventh US National Conference, edited by P. Pianetta et al. (American Institute of Physics, Melville, NY, 2000).

${ }^{7}$ Y. Kohmura, M. Awaji, Y. Suzuki, T. Ishikawa, Y. I. Dudchik, N. N. Kolchevsky, and F. F. Komarov, Rev. Sci. Instrum. 70, 4161 (1999); A. Q. R. Baron, Y. Kohmura, Y. Ohishi, and T. Ishikawa, Appl. Phys. Lett. 74, 1492 (1999).

${ }^{8}$ D. Attwood, Soft X-rays And Extreme Ultraviolet Radiation (Cambridge University Press, New York, 1999).

${ }^{9}$ V. V. Aristov, M. V. Grigoriev, S. M. Kuznetsov, L. G. Shabelnikov, V. A. Yunkin, M. Hoffman, and E. Voges, Opt. Commun. 177, 33 (2000).

${ }^{10}$ I. Snigireva et al., Proc. SPIE 4499, 64 (2001).

${ }^{11}$ For more information on this procedure, see www.ecopulse.com.

${ }^{12}$ B. Cederström, R. Cahn, M. Danielsson, M. Lundqvist, and D. Nygren, Nature (London) 404, 951 (2000); B. Cederström, Ph.D. thesis, Royal Institute of Technology, Stockholm, Sweden, 2001; see www.particle.kth.se/ $\sim$ ceder.

${ }^{13}$ E. M. Dufresne, N. R. Pereira, D. A. Arms, R. Clarke, S. B. Dierker, and D. Foster, Appl. Phys. Lett. 79, 4085 (2001); N. R. Pereira, E. M. Dufresne, D. A. Arms, R. Clarke, S. B. Dierker, and D. Foster, Proc. SPIE 4502, 173 (2001); see also www.ecopulse.com. 